\title{
THROUGHPUT GAINS OF SPACE-TIME SCHEDULING WITH NO CSI
}

\author{
Marc Realp \\ Centre Tecnològic de Telecomunicacions de Catalunya (CTTC) \\ Barcelona, Catalunya (Spain)
}

\author{
Ana I. Pérez-Neira \\ Universitat Politècnica de Catalunya (UPC) \\ Barcelona, Catalunya (Spain)
}

\begin{abstract}
Traditional works rely on the use of complete Channel State Information (CSI) at the base station to take space-time scheduling decisions in the uplink of multi-user MIMO wireless systems. However, this might be a complex and costly solution. On the other hand, the simplest scheduler is the Round Robin (RR) scheduler that schedules terminals one after the other without using CSI. However, when multiple antennas are used, many terminals could be simultaneously scheduled because of the additional spatial dimension. The optimal number of terminals to be scheduled by a space-time scheduler with no CSI in a multi-user SIMO system is investigated in this paper. Furthermore, we also study how spatial diversity and multiplexing gains are translated into throughput gains. In particular, when a ZF receiver is used, we show that the benefits of using multiple antennas increase with the minimum SNIR requirements and that, when the number of antennas is much higher than the number of terminals, the throughput gains due to multiplexing increase linearly with the number of terminals and exponentially with the minimum SNIR requirements.
\end{abstract}

\section{INTRODUCTION}

In a multi-user wireless network, the variability of the wireless channel creates different and independent channel conditions for each of the terminals (in this paper the words user and terminal are used similarly). Depending on the channel conditions some terminals might use network resources more efficiently than others. Therefore, the Multi-User Diversity (MUD) gain [1] appears. Besides, when terminals are provided with multiple antennas, the Spatial Diversity (SD) gain [2] and the multiplexing (MUX) gain [3] also play an important role. Resource allocation in MIMO systems aims at finding the optimal combination of MUD, SD and MUX gains by dynamically scheduling terminals' powers in space and time.

The optimal space-time power scheduling that maximizes the ergodic sum capacity and the capacity region in the uplink of a multi-user MIMO wireless system has been extensively investigated in [4], [5] and [6]. The general system architecture is that multiple antenna terminals communicate with a base station or access point provided also with multiple antennas. Based on the Channel State Information (CSI), the space-time scheduler at the base station decides the power to be allocated to each transmitting antenna and feeds-back such information through a feed-back channel. When the feed-back information is limited to be one scalar the best is to allocate power to only one transmitting antenna. Hence, showing the advantages

\footnotetext{
${ }^{0}$ This work was partially supported by the Catalan Government under grants SGR2005-00996 and SGR2005-00690; by the Spanish Government under projects TEC2005-08122-C03 and TEC 2006-10459/TCM; and FEDER.
}

of considering a multi-user SIMO system in limited feedback scenarios [7]. Furthermore, using a ZF receiver, the optimal space-time power scheduling policy is a policy where terminals either transmit at maximum power or do not transmit [8].

All these works rely on the use of complete CSI at the base station to take space-time scheduling decisions. However, space-time scheduling with complete CSI might be a complex and costly solution. First, because the need of instantaneous complete CSI imposes the design of specific signalling channels in order to estimate the channel state of all terminals, even when only a reduced set of terminals will be scheduled. This might reduce the effective bandwidth for data transmission. And Second, because optimal policies typically imply an exhaustive search over all the possible sets of terminals.

The simplest scheduler is the Round Robin (RR) scheduler that does not use CSI to schedule terminals but schedules terminals one after the other. However, when multiple antennas are used, many terminals could be simultaneously scheduled because of the additional spatial dimension. The optimal number of terminals to be scheduled and its relation with the system performance is something that has not been studied so far and hence, will be investigated in this paper.

In this paper we focus on analyzing the performance of space-time scheduling with no CSI in multi-user SIMO systems. We consider the average throughput as the utility function to be evaluated. Although MUD gain cannot be exploited without CSI, a space-time scheduler with no CSI can still decide how to combine MUX and SD gains. Therefore, how SD and MUX gains are translated into throughput gains is also investigated in this paper. When a $\mathrm{ZF}$ receiver is used, we show that the benefits of using multiple antennas increase with the minimum SNIR requirements and that, when the number of antennas is much higher than the number of terminals, the throughput gains due to MUX increase linearly with the number of terminals and exponentially with the minimum SNIR requirements.

\section{The Throughrut DeFinition}

Before going into the details of the space-time scheduling problem that we tackle in this paper, it is worth to devote this section to define the concept of throughput. At the PHY layer of the transmitter, data arrive from the MAC layer encapsulated into packets of $L$ bits. Such bits are encoded, modulated and transmitted through the wireless channel. We use $m$ to denote the combination of data modulation format and channel coding scheme. Then, if $R$ is the symbol transmission rate in symbols per second per Hertz, $b^{m}$ is the number of bits per symbol and $c^{m}$ is the code rate of the encoder (both, $b^{m}$ and $c^{m}$ are defined by the transmission mode $m$ ), the number of transmitted bits per second is $R b^{m} c^{m}$. Let us name $R^{m}=R b^{m} c^{m}$ as the 
effective transmission rate.

At the receiver side, the received signal is demodulated and bits are decoded and passed to the MAC layer in a packet by packet basis. Packets are received with some error probability called the Packet Error Rate (PER). In general, the PER depends on the SNR, the packet length and the transmission mode. Assuming that the SNR is constant for the whole packet transmission, we use $P E R^{m}(\gamma)$ to denote the PER when the SNR is $\gamma$ and the transmission mode is $m$. Similarly, $P S R^{m}(\gamma)=\left(1-P E R^{m}(\gamma)\right)$ denotes the Packet Success Rate (PSR). A very common and simple approach is to assume that packets are correctly received if the SNR is over a threshold value $\gamma^{m}$. That is,

$$
\operatorname{PSR}^{m}(\gamma)= \begin{cases}0 & \text { if } 0 \leq \gamma \leq \gamma^{m} \\ 1 & \text { if } \gamma>\gamma^{m}\end{cases}
$$

where $\gamma^{m}$ typically depends on the packet length $L$, and the transmission mode $m$.

Assume that a packet is transmitted at time instant $t$. Then, throughput is the amount of error-free data that the MAC layer delivers to the upper layers of the system and is given by

$$
\eta(\gamma)=R^{m} \mathbf{1}\left\{Z_{i}=1\right\} \quad[b p s / H z]
$$

where the function $\mathbf{1}\left\{Z_{i}=1\right\}$ is used as the indicator function of a Bernoulli distributed random variable $Z_{i}$ that takes value 1 with probability $P S R^{m}(\gamma)$.

Note that throughput is a random variable that varies in time, even when channel conditions, given by $\gamma$, are constant. Therefore, the expected throughput when the SNR is $\gamma$ is defined as

$$
\bar{\eta}(\gamma)=E_{t}\{\eta(\gamma)\}=R^{m} P S R^{m}(\gamma)[b p s / H z]
$$

and the average throughput is obtained averaging over the channel fluctuations

$$
\bar{\eta}=E_{\gamma}\left\{R^{m} P S R^{m}(\gamma)\right\} \quad[b p s / H z]
$$

\section{Formulation of THE SPACE-Time Scheduling PROBLEM}

Let us consider a SIMO multiple access channel where $N$ terminals with one antenna each communicate with the RT (Receiver Terminal), provided with $M$ antennas. Assume a block fading channel such that a channel realization $\mathbf{H}$ is constant for a packet transmission. Previous to the transmission of packets, a set $\mathcal{K}$ of active terminals (all transmitting at maximum individual powers) receive access to the channel through the feedback channel. We assume a scalar feedback channel that is ideal and error-free.

Our space-time scheduling policy is defined by a resource allocation vector $\mathbf{p}=\left\{p_{\mathcal{K}}: \mathcal{K} \subseteq \mathcal{P}\{1, . ., N\}\right\}^{1}$, with $p_{\mathcal{K}} \in$ $[0,1]$ and $\sum_{\mathcal{K}} p_{\mathcal{K}} \leq 1$ where $p_{\mathcal{K}}$ is the probability that terminals in set $\mathcal{K}$ are scheduled. In other words, $\mathbf{p}$ is a time-sharing policy among different sets of terminals. Assume that $p_{\mathcal{K}}=1$ and $p_{\mathcal{K}^{\prime}}=0$ for $\mathcal{K}^{\prime} \neq \mathcal{K}$. Hence, the subset of terminals $\mathcal{K}$ transmit simultaneously with probability one. We denote the

\footnotetext{
${ }^{1}$ We use $\mathcal{P}\{1, . ., N\}$ to indicate all the partitions of the set $\{1, . ., N\}$
}

cardinality of $\mathcal{K}$ by $\mathbf{K}$. The following is a model for this multiaccess antenna-array communication link

$$
\mathbf{y}_{\mathcal{K}}=\mathbf{H}_{\mathcal{K}} \mathbf{s}_{\mathcal{K}}+\mathbf{w}_{\mathcal{K}}
$$

The vector $\mathbf{s}_{\mathcal{K}}=\left(s_{1}, s_{2}, . ., s_{\mathbf{K}}\right)^{T}$ is the transmitted symbol vector where $s_{n}$ is the transmitted symbol of the $n$th terminal in $\mathcal{K}, \mathbf{H}_{\mathcal{K}}=\left(\mathbf{h}_{1}, \mathbf{h}_{2}, \ldots, \mathbf{h}_{\mathbf{K}}\right)$ is the $M \times \mathbf{K}$ flat-fading channel matrix where the scalar $h_{m, n}$ represents the fading suffered by the $n$th transmitter in the set $\mathcal{K}$ at the $m$ th receiver antenna. Note that $\mathbf{H}_{\mathcal{K}}$ is a matrix that gathers the columns in $\mathbf{H}$ corresponding to the terminals in the set $\mathcal{K}$. The vector $\mathbf{w}_{\mathcal{K}}$ is a complex-valued, background Gaussian noise with zero mean and variance $\sigma_{w}^{2}$. Then, given a channel realization $\mathbf{H}$ (which in turn defines $\mathbf{H}_{\mathcal{K}}$ ), the instantaneous SNIR for the $n$th terminal in $\mathcal{K}$ is defined as

$$
\gamma_{n}=\bar{\gamma} \alpha_{n}\left(\mathbf{H}_{\mathcal{K}}\right)
$$

where $\bar{\gamma}=\frac{p}{\sigma_{w}^{2}}$ is the average SNR and $\alpha_{n}\left(\mathbf{H}_{\mathcal{K}}\right)$ its SNIR enhancement factor. The SNIR enhancement factor $\alpha_{n}\left(\mathbf{H}_{\mathcal{K}}\right)$ can be seen as a quality measurement that accounts for the effect of channel fading and Multiple Access Interference (MAI) on the received SNR through the receiver implementation. Many models could be developed for $\alpha_{n}\left(\mathbf{H}_{\mathcal{K}}\right)$ depending on the receiver that one wants to take into account [9], [10]. To emphasize the dependence on $\alpha_{n}\left(\mathbf{H}_{\mathcal{K}}\right)$, we rewrite the $n$th terminal's PSR as $P S R^{m}\left(\alpha_{n}\left(\mathbf{H}_{\mathcal{K}}\right)\right)$.

Because resource allocation decisions are taken without using CSI, the individual average throughput is

$$
\bar{\eta}_{n}=\sum_{\substack{\mathcal{K} \subseteq \mathcal{P}\{1, \ldots, N\} \\ \text { s.t. } n \in \mathcal{K}}} p_{\mathcal{K}} E_{\mathbf{H}}\left\{R^{m} P S R^{m}\left(\alpha_{n}\left(\mathbf{H}_{\mathcal{K}}\right)\right)\right\}
$$

and the total average throughput is $\bar{\eta}=\sum_{n} \bar{\eta}_{n}$.

Let us present the SD and MUX gains in this context. Assume that $p_{\mathcal{K}}=1$ and $p_{\mathcal{K}^{\prime}}=0$ for $\mathcal{K}^{\prime} \neq \mathcal{K}$. Because all the antennas are devoted to the transmission of many terminals, we say that the MUX gain is exploited. In that case, the individual average throughput is given by

$$
\bar{\eta}_{n}^{M U X}= \begin{cases}0 & \text { if } n \notin \mathcal{K} \\ E_{\mathbf{H}}\left\{R^{m} P S R^{m}\left(\alpha_{n}\left(\mathbf{H}_{\mathcal{K}}\right)\right)\right\} & \text { if } n \in \mathcal{K}\end{cases}
$$

Given a set of terminals $\mathcal{K}$, we define the MUX gain $G_{\mathcal{K}}^{M U X}$ as the proportional increase in the total average throughput with respect to the total average throughput in the single user SISO case, i.e., when $\mathcal{K}=\{n\}$ and $M=1$. The single-user SISO average throughput is $\bar{R}^{m}=E_{\mathbf{H}}\left\{R^{m} P S R^{m}\left(\alpha_{n}\left(h_{n}\right)\right)\right\}$ where, in that case, the channel matrix $\mathbf{H}_{\mathcal{K}}$ is a scalar $h_{n}$. Then, the MUX gain is given by,

$$
G_{\mathcal{K}}^{M U X}=\frac{\sum_{n} \bar{\eta}_{n}^{M U X}}{\bar{R}^{m}}=\frac{\sum_{n \in \mathcal{K}} \bar{\eta}_{n}^{M U X}}{\bar{R}^{m}}
$$

Assume that all terminals use the same transmission mode $m$. Then, because all terminals behave similarly in the 
average, the expectation $E_{\mathbf{H}}\left\{R^{m} P S R^{m}\left(\alpha_{n}\left(\mathbf{H}_{\mathcal{K}}\right)\right)\right\}$ is the same for any $n \in \mathcal{K}$ and for any $\mathcal{K}$ with cardinality $\mathbf{K}$. That is formally expressed as $E_{\mathbf{H}}\left\{R^{m} P S R^{m}\left(\alpha_{n}\left(\mathbf{H}_{\mathcal{K}}\right)\right)\right\}=$ $E_{\mathbf{H}}\left\{R^{m} P S R^{m}\left(\alpha_{n^{\prime}}\left(\mathbf{H}_{\mathcal{K}^{\prime}}\right)\right)\right\} \forall n \in \mathcal{K}, n^{\prime} \in \mathcal{K}^{\prime}$ and $|\mathcal{K}|=\left|\mathcal{K}^{\prime}\right|=\mathbf{K}$. Therefore, the equality $G_{\mathcal{K}}^{M U X}=G_{\mathcal{K}^{\prime}}^{M U X}=$ $G_{\mathrm{K}}^{M U X}$ is true with

$$
\begin{aligned}
G_{\mathbf{K}}^{M U X} & =\mathbf{K} \frac{E_{\mathbf{H}}\left\{R^{m} P S R^{m}\left(\alpha_{n}\left(\mathbf{H}_{\mathcal{K}}\right)\right)\right\}}{\bar{R}^{m}} \\
& =\mathbf{K} \frac{E_{\mathbf{H}}\left\{R^{m} P S R^{m}\left(\alpha_{n^{\prime}}\left(\mathbf{H}_{\mathcal{K}^{\prime}}\right)\right)\right\}}{\bar{R}^{m}}
\end{aligned}
$$

In consequence, $G_{\mathbf{K}}^{M U X}$ describes the gain on the total average throughput when terminals are space-time scheduled in groups of $\mathbf{K}$ with independence of what is the set $\mathcal{K}$ that is chosen every-time. That is, $G_{\mathrm{K}}^{M U X}$ is the MUX gain when $\sum_{\mathcal{K} \text { s.t. }|\mathcal{K}|=\mathbf{K}} p_{\mathcal{K}}=1$. Recall that $G_{\mathbf{K}}^{M U X}$ is the total average throughput gain with respect to the total average throughput in the single user SISO case. Hence, accounts for the benefits of using multiple antennas and having multiple users in the system. When $\mathcal{K}=\{n\}$, all the antennas are devoted to the transmission of terminal $n$ and then, the SD gain is fully exploited. Therefore, we define the SD gain as $G^{S D}=G_{1}^{M U X}$

One objective of the designer would be to obtain the optimal value of $\mathbf{K}^{*}$ such that

$$
\mathbf{K}^{*}=\arg \max _{\mathbf{K}} G_{\mathbf{K}}^{M U X}
$$

and therefore, to achieve the maximum throughput gain by scheduling terminals in groups of $\mathbf{K}^{*}$. A special case is when $\mathbf{K}^{*}=1$, i.e. when $G_{1}^{M U X}>G_{\mathbf{K}}^{M U X}$ for $\mathbf{K}=2, \ldots, M$. Since $G^{S D}=G_{1}^{M U X}$, this means that spatial diversity is better than multiplexing and therefore, the maximum average throughput gain is achieved by scheduling terminals in groups of 1 , i.e. performing a RR scheduling or TDMA.

Alternatively, the objective could be to find at least one of all the possible values of $\mathbf{K}$ such that, $G_{\mathbf{K}}^{M U X}>N$. In that case, the total average throughput is multiplied by a factor higher than the number of terminals in the system meaning that, in the multi-user SIMO system, $N$ terminals can be served with an average throughput equal or higher than that in the single-user SISO case.

Recall that $G_{\mathbf{K}}^{M U X}$ is achieved when $\sum_{\mathcal{K} \text { s.t. }|\mathcal{K}|=\mathbf{K}} p_{\mathcal{K}}=1$. However, the policy $\mathbf{p}$ with the general restriction of $\sum_{\mathcal{K}} p_{\mathcal{K}} \leq$ 1 is the one that governs how the different multiplexing gains are distributed among the terminals. In other words, by means of the policy $\mathbf{p}$, the scheduler could decide how often to schedule a set of terminals $\mathcal{K}$. Therefore, giving more or less throughput to different terminals. Let us define the individual throughput gain as

$$
G_{n}=\frac{\bar{\eta}_{n}}{\bar{R}^{m}}=\sum_{\substack{\mathcal{K} \subseteq \mathcal{P}\{1, \ldots, N\} \\ \text { s.t. } n \in \mathcal{K}}} p_{\mathcal{K}} \frac{G_{\mathbf{K}}^{M U X}}{\mathbf{K}}
$$

and the gain vector $\mathbf{G}$ as $\mathbf{G}=\left(G_{1}, \ldots, G_{N}\right)$. Then, the gain region $\Phi$ is the defined as the vector set of all achievable gain vectors and is expressed as

$$
\Phi=\cup_{\mathbf{p} \in \mathcal{P}}\left\{\mathbf{G} \in \mathbb{R}^{N}: G_{n} \mid p_{\mathcal{K}} \in[0,1], \sum_{\mathcal{K}} p_{\mathcal{K}} \leq 1\right\}
$$

With some abuse of notation, $\mathcal{P}$ is the set of all possible policies p. Since $p_{\mathcal{K}} \frac{G_{\mathrm{K}}^{M U X}}{\mathrm{~K}}$ increases with respect to $p_{\mathcal{K}}$, the gain region is convex. Therefore, the boundary of $\Phi$, denoted by $\partial \Phi$, is the convex upper envelope of the points that are the solution to the maximization of the sum of prioritized individual gains for a priority vector $\boldsymbol{\theta}=\left\{\theta_{\{1\}}, \ldots, \theta_{\mathcal{K}}, \ldots, \theta_{\{1, \ldots, N\}}\right\} \in \mathbb{R}_{+}^{\mathbf{N}}$ being $\theta_{\mathcal{K}}$ the priority given to the set of terminals $\mathcal{K}$ and $\mathbf{N}$ the total number of possible sets. That is

$$
\max _{\mathbf{p}}\left\{\sum_{n} \sum_{\substack{\mathcal{K} \subseteq \mathcal{P}\{1, \ldots, N\} \\ \text { s.t. } n \in \mathcal{K}}} p_{\mathcal{K}} \theta_{\mathcal{K}} \frac{G_{\mathbf{K}}^{M U X}}{\mathbf{K}}\right\}
$$

with solution given by the policy

$p_{\mathcal{K}}^{*}(\boldsymbol{\theta})=\left\{\begin{array}{c}1 \quad \text { if } \mathcal{K}=\arg \max _{\mathcal{K}^{\prime} \subseteq \mathcal{P}\{1, . ., N\}} \theta_{\mathcal{K}^{\prime}} \sum_{n \in \mathcal{K}^{\prime}} \frac{G_{\mathbf{K}^{\prime}}^{M U X}}{\mathbf{K}^{\prime}} \\ 0 \quad \text { if } \mathcal{K} \neq \arg \max _{\mathcal{K}^{\prime} \subseteq \mathcal{P}\{1, . ., N\}} \theta_{\mathcal{K}^{\prime}} \sum_{n \in \mathcal{K}^{\prime}} \frac{G_{\mathbf{K}^{\prime}}^{M U X}}{\mathbf{K}^{\prime}}\end{array}\right.$

where $\mathbf{K}^{\prime}$ is the cardinality of $\mathcal{K}^{\prime}$. Equivalently,

$$
p_{\mathcal{K}}^{*}(\boldsymbol{\theta})= \begin{cases}1 & \text { if } \mathcal{K}=\arg \underset{\max _{\mathcal{K}^{\prime} \subseteq \mathcal{P}\{1, . ., N\}}}{ } \theta_{\mathcal{K}^{\prime}} G_{\mathbf{K}^{\prime}}^{M U X} \\ 0 & \text { if } \mathcal{K} \neq \arg \underset{\max _{\mathcal{K}^{\prime} \subseteq \mathcal{P}\{1, . ., N\}}}{ } \theta_{\mathcal{K}^{\prime}} G_{\mathbf{K}^{\prime}}^{M U X}\end{cases}
$$

We used the notation $p_{\mathcal{K}}^{*}(\boldsymbol{\theta})$ instead of $p_{\mathcal{K}}$ to emphasize that the set of terminals $\mathcal{K}$ is scheduled with probability 1 or 0 depending on the priority vector $\boldsymbol{\theta}$. Let $G_{n}(\boldsymbol{\theta})$ denote the individual gain when $p_{\mathcal{K}}^{*}(\boldsymbol{\theta})$ is the solution of (16). Then, the gain region boundary $\partial \Phi$ is the convex upper envelope of the points $\left\{G_{n}(\boldsymbol{\theta}): \boldsymbol{\theta} \in \mathbb{R}_{+}^{N}, \sum \theta_{\mathcal{K}}=1\right\}$. Because of the convex envelope opperation, it can be easily observed that any point at the boundary is obtained by combining different $p_{\mathcal{K}}^{*}(\boldsymbol{\theta})$ in time (time-sharing).

\section{AN EXAMPLE WITH A ZF RECEIVER}

In this section, we present an example on how to compute the multiple antenna gains and the gain region in space-time scheduling with no CSI. Assume the use of a $\mathrm{ZF}$ receiver and define the SNIR enhancement factor $\alpha_{n}\left(\mathbf{H}_{\mathcal{K}}\right)$ as $\alpha$. Being the entries of $\mathbf{H}_{\mathcal{K}}$ independent and identically distributed Gaussian random variables with zero mean and unit variance (i.e. Rayleigh fading), the random variable $\alpha$ is a weightened Chi-Square distributed variable with $2(M-\mathbf{K}+1)$ degrees of freedom and p.d.f. denoted by $f_{\mathbf{K}}(\alpha)$. Furthermore, from (1) and (6) we define $\alpha^{m}=\frac{\gamma^{m}}{\bar{\gamma}}$ as the minimum SNIR enhancement factor for which packets are accepted by the upper layers of the communication system.

Combining (1) with the p.d.f. of $\alpha$, the average throughput due to MUX gain is ${ }^{2}$

\footnotetext{
${ }^{2}$ The average throughput is computed assuming that $R^{m}$ is constant, i.e., that no link adaptation is performed.
} 


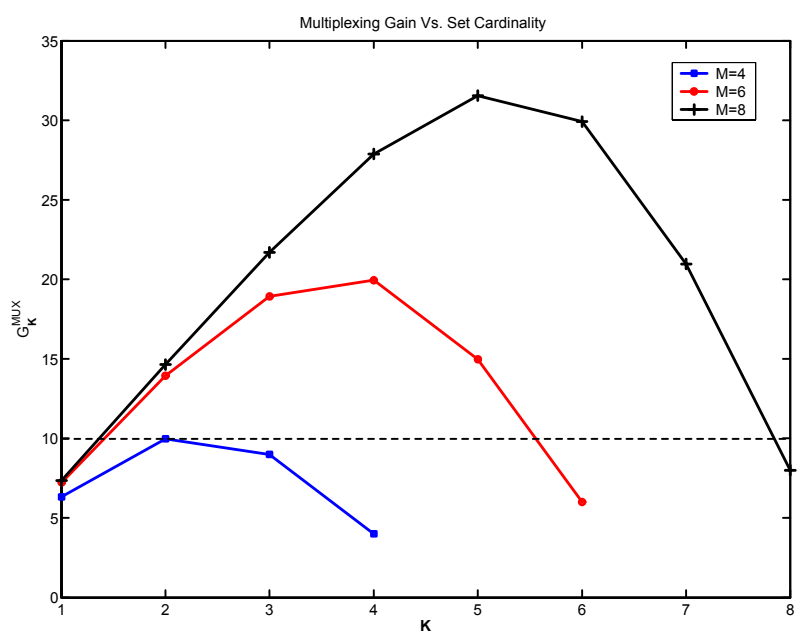

Figure 1: MUX Gain Vs. the cardinality of $\mathcal{K}$. A Zero Forcing receiver and the QoS parameter $\alpha^{m}$ is $3 d B$. if $n \notin \mathcal{K}$
if $n \in \mathcal{K}$

where $\Gamma(\alpha, x)$ is $\int_{x}^{\infty} e^{-t} t^{\alpha-1} d t$. Therefore, the multiplexing gain is easily computed as

$$
G_{\mathbf{K}}^{M U X}=\mathbf{K} \frac{\frac{\Gamma\left(M-\mathbf{K}+1, \alpha^{m}\right)}{(M-\mathbf{K}) !}}{\Gamma\left(1, \alpha^{m}\right)}=\mathbf{K} \sum_{i=0}^{M-\mathbf{K}} \frac{\left(\alpha^{m}\right)^{i}}{i !}
$$

and the maximum MUX gain is given by (11).

From (18), we observe that the MUX gain increases with $\alpha^{m}$. Therefore, the advantage of using multiple antennas increases with the minimum SNIR requirements. Furthermore, if we consider that the number of receiver antennas is high compared to the number of terminals, we obtain the upper bound

$$
G_{\mathbf{K}}^{M U X} \leq \lim _{M \rightarrow \infty} \mathbf{K} \sum_{i=0}^{M-\mathbf{K}} \frac{\left(\alpha^{m}\right)^{i}}{i !}=\mathbf{K} e^{\alpha^{m}}
$$

Hence, in the limit the MUX gain increases exponentially with the minimum SNIR and linearly with the number of terminals.

From (18), we observe that the multiplexing gain depends on the number of antennas at the $\mathrm{ZF}$ receiver $M$, the number of terminals that are scheduled $\mathbf{K}$, and the minimum SNIR enhancement for which packets are accepted by the upper layers of the communication system $\alpha^{m}$. Now, assume, for instance, a video streaming application with a minimum SNIR requirement of $\alpha^{m}$ and also assume that the system parameters (the effective transmission rate $R^{m}$, the average SNR, etc) are such that the average throughput in the single user SISO case (see for instance, (17) with $M=\mathbf{K}=1$ ) is satisfactory for the application. Then, a MUX gain of $G_{\mathbf{K}}^{M U X}$ indicates that, by using $M$ antennas and scheduling terminals in groups of $\mathbf{K}$, an equivalent of $G_{\mathbf{K}}^{M U X}$ video streams with minimum SNIR requirement

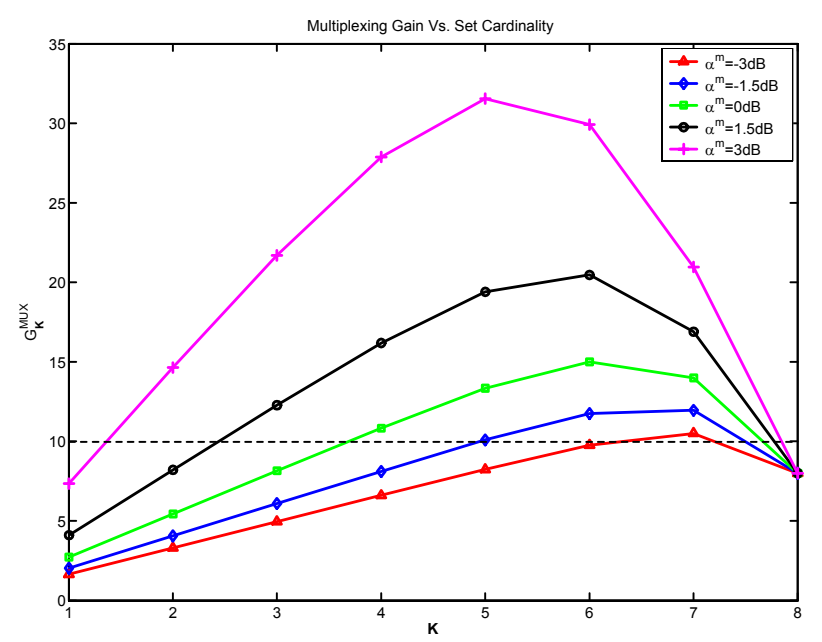

Figure 2: MUX Gain Vs. the cardinality of $\mathcal{K}$. A Zero Forcing receiver is used with $M=8$ antennas.

$\alpha^{m}$ and effective transmission rate $R^{m}$ can be supported. That is, the total average throughput is multiplied by $G_{\mathbf{K}}^{M U X}$.

In figure 1, the multiplexing gain is plotted as a function of $\mathbf{K}$ and $M$ when $\alpha^{m}$ is fixed. The optimal $\mathbf{K}^{*}$ that maximizes $G_{\mathbf{K}}^{M U X}$ increases with $M$. Note that the optimal $\mathbf{K}^{*}$ maximizes the multiplexing gain and hence, the total average throughput of the system. Furthermore, we observe that the same multiplexing gain can be achieved by different combinations of $\mathbf{K}$ and $M$. This is interesting because, $\mathbf{K}$ is intrinsically related to the average access delay. Indeed, the higher the $\mathbf{K}$, the more the number of terminals that transmit in a time-slot and therefore, the less the time that a terminal should wait to get access to the channel. Clearly, the average access delay is minimized by $\mathbf{K}=M$. In that case, the multiplexing gain is aleays equal to $M$. Then, a trade-off between access delay and MUX gain appears.

In figure 2, the $G_{\mathbf{K}}^{M U X}$ is presented as a function of $\mathbf{K}$ and $\alpha^{m}$ when $M$ is fixed. For each value of $\alpha^{m}$, there exists an optimal $\mathbf{K}^{*}$ that maximizes $G_{\mathbf{K}}^{M U X}$. We observe that as $\alpha^{m}$ increases, the optimal $\mathbf{K}^{*}$ decreases meaning that the more stringent the minimum SNIR requirements are, the more the access delay a terminal must expect. Furthermore, for the same number of antennas and the same set cardinality, the multiplexing gain increases with $\alpha^{m}$ indicating that the benefits of using multiple antennas increase with the SNIR requirements. Note however, that the multiplexing gain is the gain with respect to the single user SISO case. Then, systems with different $\alpha^{m}$ requirements have different single-user SISO average throughputs and therefore, a system with higher multiplexing gain does not necessarily mean a system with a higher total average throughput. In figure $3, G_{\mathrm{K}}^{M U X}$ is presented as a function of $\alpha^{m}$ and $M$ when $\mathbf{K}$ is fixed. As we could expecte from (18), we observe that when $M>\mathbf{K}$, the MUX gain increases with $\alpha^{m}$ and approaches the upper bound (19).

The gain region (13) is easily computed by using (16) and (18). In figure 4 the gain region for different values of $\alpha^{m}$ is 


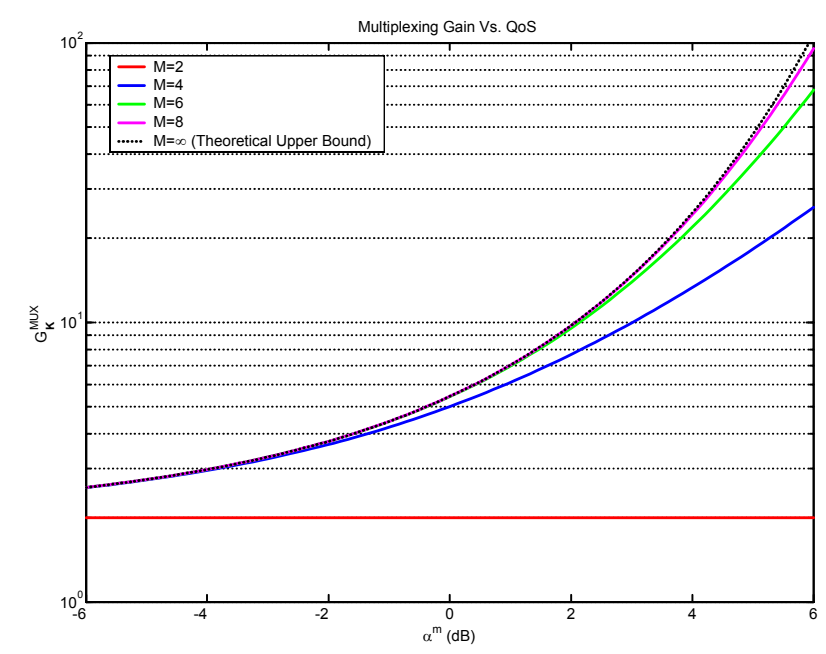

Figure 3: MUX Gain Vs. the QoS parameter $\alpha^{m}$. K is fixed to 2.

presented. In general, the gain region is a polytope. In the two terminal space, such polytope is a polygon where the upper right vertex corresponds to the individual gains when the MUX gain is fully exploited, that is, when $\theta_{\{1,2\}}=1$ and hence, $p_{\{1,2\}}^{*}(\boldsymbol{\theta})=1$. In that case, such vertex is the point $\mathbf{G}=\left(G_{1}, G_{2}\right)=\left(\frac{G_{2}^{M U X}}{2}, \frac{G_{2}^{M U X}}{2}\right)$. The lower right vertex and the higher left vertex, are the points $\left(G_{1}, 0\right)=\left(G_{1}^{M U X}, 0\right)$ and $\left(0, G_{2}\right)=\left(0, G_{1}^{M U X}\right)$ respectively, i.e. when $\theta_{\{1\}}=1$ and $\theta_{\{2\}}=1$ respectively, and correspond to the individual gains when antennas are devoted to fully exploit the SD gain. Recall that $G^{S D}=G_{1}^{M U X}$. The gain region boundary is obtained by time sharing between these three points. Therefore, the average throughput region is a polygon as long as $G_{2}^{M U X}>G_{1}^{M U X}$. If this is the case there is a benefit of multiplexing the two terminals simultaneously. However, when $G_{2}^{M U X}<G_{1}^{M U X}$, there is no multiplexing benefit and is always better to schedule the two terminals separately, i.e., to perform a pure TDMA policy. In that case, the average throughput region becomes a triangle instead of a polygon. In figure 4 this occurs when $\alpha^{m} \geqslant 0 d B$.

\section{CONCLUSIONS}

Due to the fact that resource allocation with CSI might be a costly solution, how to schedule terminals in the uplink of a multi-user SIMO scenario without using CSI was studied in this paper. One of the novelties of the paper was to evaluate system performance in terms of throughput (a MAC layer figure of merit) that allows to analyze the effects of QoS requirements such as the minimum SNIR. By investigating the SD and MUX gains when a $\mathrm{ZF}$ receiver is used, we found out that there exists an interesting trade-off between multiple antenna gains (MUX and SD) and QoS requirements. In particular, we showed that the average throughput gains are related to the number of antennas used, the number of terminals that are scheduled and the minimum SNIR requirements. The multiple antenna benefits increase with the minimum SNIR requirements and in the limit,

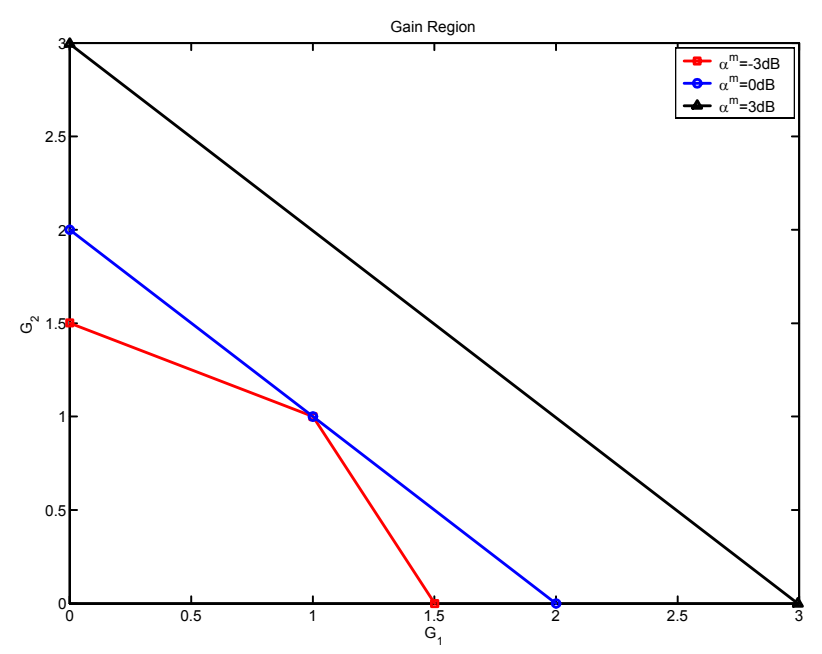

Figure 4: Gain Region for a system with two transmitting terminals and one RT. A two antenna $(M=2) \mathrm{ZF}$ receiver is used.

the MUX gain increases linearly with the number of scheduled terminals and exponentially with the SNIR requirements.

\section{REFERENCES}

[1] R. Knopp and P. A. Humlet, "Information Capacity and Power Control in Single-Cell Multiuser Communications", in Proc IEEE ICC 1995, June 1995.

[2] S. Alamouti, "A simple transmitter diversity scheme for wireless communications", IEEE Journal on Select. Areas Commun., Vol. 16, Page(s): 1451-1458, October 1998.

[3] P.W. Wolniansky, G.J Foschini, G.D. Golden, and R.A. Valenzuela, "VBLAST: An architecture for realizing very high data rates over the rich scattering wireless channel", in Proc. ISSSE 98, September 1998.

[4] W. Yu, W. Rhee and J. M. Cioff, "Optimal Power Control in Multiple Access Fading Channels", in Proc. IEEE ICC 2001.

[5] W. Yu, W. Rhee, S. Boyd, and J. M. Cioffi, "Iterative water-filling for Gaussian vector multiple access channels", IEEE Trans. on Information Theory, Vol. 50, Page(s): 145-151, January 2004.

[6] W.Rhee and J. M.Cioffi . On the capacity of multiuser wireless systems with multiple antennas IEEE Trans. on Information Theory, Vol. 49, Page(s): 2580-2595, October 2003.

[7] V. K. N. Lau, Y. Liu, T-A Chen, "Optimal Space-Time Scheduling for Block Fading Channels with Partial Power Feedbacks", Bell Labs Technical Journal 7 (3),Page(s): 27-46, 2003.

[8] V. K. N. Lau, Y.-K. Kwok, "Performance Analysis of SIMO Space-Time Scheduling with Convex Utility Function: Zero-Forcing Linear Processing", IEEE Trans. on Vehicular Technology, Vol. 53, NO. 2, Page(s): 339-350, March 2004.

[9] M. Realp and A. I. Pérez-Neira, "Generalized Model for Scheduling in MIMO Multiple Access Systems: A Cross-Layer Approach", Elsevier Sig. Proc. journal, Vol. 86, Issue 8, Page(s): 1834 - 1847, August 2006

[10] N. Wang and S. D. Blostein, "Approximate Minimum BER Power Allocation for MIMO Spatial Multiplexing Systems", IEEE Trans. on Communications, Vol. 55, NO. 1, Page(s): 180-187 January 2007 